EDITORIAL

\title{
AN ORGANIZING MODEL FOR THOSE ENTERING THE FIELD OF PSYCHIATRY
}

\author{
LLOYD I. SEDERER, M.D.
}

\begin{abstract}
To enter the field of psychiatry is to encounter an overwhelming mass of clinical data, hypothetical notions, and theoretical constructs. Conceptual models are tools by which the trainee begins to master the field and these complexities. For psychiatry, the biopsychosocial model has become the prevailing conceptual model (and DSM III R its specific nosology). This paper will offer a method for the application of the biopsychosocial model in order to demonstrate its utility and diminish problems in its utilization.
\end{abstract}

\section{Introduction}

To set foot into the field of psychiatry is to encounter an overwhelming mass of clinical data, hypothetical notions, and theoretical constructs. Dopamine mingles with denial, and serotonin with symbiosis. Defenses and divorce appear as meaningful, and influential, as gamma aminobutyric acid and the endorphins. Urban drift, ego-deficits, and ventricular enlargement may be found rubbing conceptual shoulders.

To then march ahead and begin to practice psychiatry is to assume the responsibilities inherent to the profession, and the liabilities therein. In a recent troubling lawsuit a patient who had been hospitalized at a private psychiatric hospital successfully sued that facility for negligence on the basis of having been given a psychodynamic diagnosis (narcissistic personality disorder) and respective treatment plan, rather than having been diagnosed as having a major depression and given appropriate somatic treatment. ${ }^{1}$ How available data is organized conceptually is no longer simply an academic or clinical matter; it is a matter of professional competence or malpractice, as the case may be.

Address reprint requests to: Lloyd I. Sederer, M.D., Mount Auburn Hospital, Harvard Medical School, 330 Mount Auburn Street, Cambridge, Massachusetts 02238. The author would like to thank Drs. Jane Thorbeck, Howard Shaffer and Robert Drake for their contributions to this manuscript. 
Carefully made diagnoses, furthermore, create treatment dilemmas. There is no such thing as an innocuous treatment intervention. Benefit must be weighed against risk. The sequence of interventions must consider potential for remedy as well as how invasive the treatment, and treatment setting, may be. Electroconvulsive therapy may be highly efficacious, but it is also invasive. Involuntary treatment has become so legally constrained in some states as to seriously limit appropriate care for acutely ill, involuntary hospitalized psychiatric patients. ${ }^{2}$ Any disorder warranting invasive treatment or a restrictive environment must be distinguished from lesser problems, and substantiated diagnostically and clinically, in order to medico-legally support certain interventions.

This paper will offer an organizing model for those entering the field of psychiatry. In order to wind his way through the clinical thicket of data, diagnosis, and treatment, the trainee requires a model that is first comprehensive. The model must then allow him to proceed in an orderly manner to develop clinical hypotheses (hunches) ${ }^{3}$ that lead to hierarchically organized diagnoses and sequentially organized treatments. Finally, the model itself must make common sense and fit within basic understandings of human nature that the trainee has already incorporated intuitively in the years preceeding formal training.

\section{The Biopsychosocial Model}

The practice of clinical psychiatry draws its conceptual underpinnings from the amalgam of biological, psychological, and social perspectives which we generally refer to as the biopsychosocial model. ${ }^{4-6}$ Each perspective alone represents a particular bias worthy of our inspection.

The biological perspective is a disease orientation. The medical model perhaps best represents this prescription. People are hosts of disease entities, containers for discreet pathogenic agents or exponents of heriditable maladaptions. A cluster of signs and symptoms, valid and reliable, defines each disorder, which has a distinct pathogenesis, course, and prognosis. If the etiology is not known, it is the focus of research efforts. Treatment is specific to the etiology, or the symptom cluster, and is aimed at cure or palliation.

Psychology offers a considerably different cast of mind in which developmental difficulties, intrapsychic conflict, disturbed relationships, and learned maladaptive thoughts and behaviors generate personality disorder and personal distress. Personality profiles and disordered behaviors, rather than pathogens and symptoms, determine diagnosis. Etiology is inferred and treatment is rooted in the doctor-patient relationship (unlike the medical model where the doctor-patient relationship is an 
enabling factor, rather than a curative factor). The goal of medical cure is replaced by psychological goals of adaptation, and the ineffable, wornthin, and almost unquantifiable notion of growth.

The social perspective requires that we step out of the person's biology and psychology, in order to appreciate his context or environment. A variety of matters must be examined because a person is considered in dynamic equilibrium with his environment. Crises in the family, vocational misfortunes, environmental dislocations and disturbances (e.g. forced emigration, geological catastrophes, governmental failures) may disturb the fragile equilibrium of any individual and prompt symptomatology. Treatment is not specific to the person, rather it is aimed at specific environmental interventions. Success is measured by symptom diminution, and notions like cure and personal growth are conceptual misnomers.

The biopsychosocial model offers the modern practitioner a synthesis of these three prescriptions. Widely disparate, and potentially competing, prescriptions are merged. Disease, person and social situation converge in a manner fully reminiscent of Voltaire and his Holy Trinity of Destiny, in which heredity, environment and circumstance were the perceived agents of our fate. Though this model falls short of a unifying and organizing paradigm, it does provide a broad and coherent conceptual framework. ${ }^{7-10}$

\section{Application of The Biopsychosocial Model}

The biopsychosocial model can be represented on a three dimensional graphic, in which Axis A is biological, Axis B is psychological, and Axis C is social.

Each axis represents one dimension of the person before us, who has come in search of help. Not only is each axis a distinct perspective; the degree of disorder on each axis can be weighed and graphed. Figure 1 is a three dimensional representation of the biopsychosocial model, with each axis quantified according to three degrees of disturbance (minimal, moderate, severe). For example, an individual's biology (which would be graphed on Axis A) could evidence minimal, moderate, or severe symptomatology; the person's psychology, or character or personality disorder*; and the person's social circumstances (Axis C) could also be

\footnotetext{
${ }^{*}$ The reader is especially referred to Kernberg ${ }^{11,12}$ and Vaillant ${ }^{13}$ for systems of hierarchizing personality levels and organization. Kernberg has provided a schemata for understanding personality organization from high to mid-and low levels of organization, which is derived from an assessment of ego functions (defenses, adaptation, and object relations); superego construction; and the nature of the individual's instincts. Vaillant has offered a hierarchy of defenses as a methodology of assessing personality development.
} 
roughly quantified according to degree of disorder. The center point of the graph represents an ideal state of health, personality organization, and social stability and resource-richness.

\section{FIGURE 1}

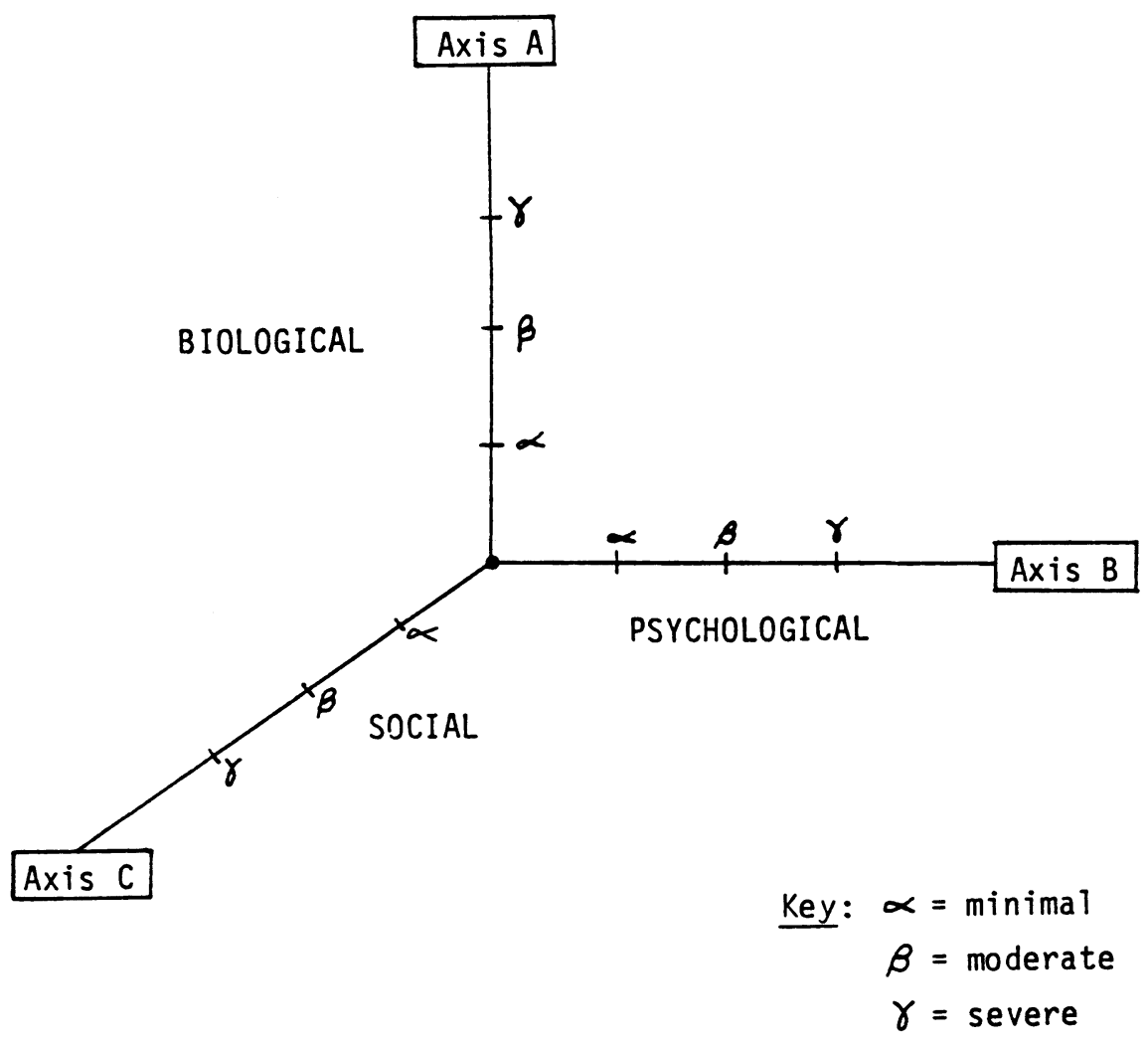

Psychiatry's biological perspective is mapped on Axis A; it is also organized in DSM III R on its Axis I. This axis, for both the biopsychosocial model and DSM III R, contains psychiatry's "diseases": mood, thought, and certain discreet symptomatic disorders (e.g., anorexia nervosa) are contained within this axis. This is not to say that a biological etiology exists or does not exist for these disorders; rather it is to 
identify them as the "diseases" of psychiatry, each with its specific symptom constellation, pathogenesis, course and prognosis. I am not implying that this is the only way of viewing these disorders; rather, I am simply using a convention, with its limitations and values. Axis B on our model is the psychological perspective and, as such, is the depiction of the patient's personality or character. Axis B fits neatly with Axis II of DSM III R, which lists a variety of personality types, or in more severe forms, disorders. Examples include obsessive-compulsive, histrionic, narcissistic, borderline, and sociopathic personality configurations. Axis $\mathrm{C}$, the social perspective of the biopsychosocial model, departs radically from Axis III of DSM III $R$, which is a medical illness classification. Axis IV and V of DSM III R describe psychosocial stressors and level of adaptation. In the biopsychosocial model, Axis $\mathrm{C}$ depicts a range of social considerations, including the social milieu of family and friends; finances; education, ethnicity, and attitudes toward health care. With the zero point on Axis $\mathrm{C}$ marking a state of social stability and resourcerichness, disturbances and deficiencies in the patient's social field can be quantified as being from minimally disturbed and resource-depleted to severely disturbed and resource-exhausted.

By using the disorder of major depression as it occurs in two quite distinct patients and social circumstances, we can now apply the model and bring it down to the everyday earth of clinical practice.

\section{Case \#1}

Dr. $\mathrm{M}$ is a 53-year old university professor who sought out-patient psychiatric consultation for the first time in her life for increasing depressive symptomatology of several months duration. Not psychotic, she did meet virtually all the DSM III R criteria for major depression, and demonstrated severe neurovegetative symptomatology. Her family history was positive for affective disorder in the maternal lineage and was negative on both sides for alcoholism and severe personality disorder. Psychologically she was a woman of obsessive manner; she was married, with two children, and stable and maintained long-lasting friendships. Though affectively constricted, she had made methodical adaptations throughout her years and had built a rewarding personal and professional life. In the past year, Dr. M's mother had become progressively ill with cardiac disease and an area of Dr. M's research work was in jeopardy because of loss of federal funding. Nevertheless, her family was stable, supportive, available, and had the financial reserves to tolerate any loss of work that Dr. M. might have because of depression. Despite feelings of depressive hopelessness, Dr. M. did respect the field of psychiatry and was able to ally with appropriate care. 


\section{FIGURE 2}

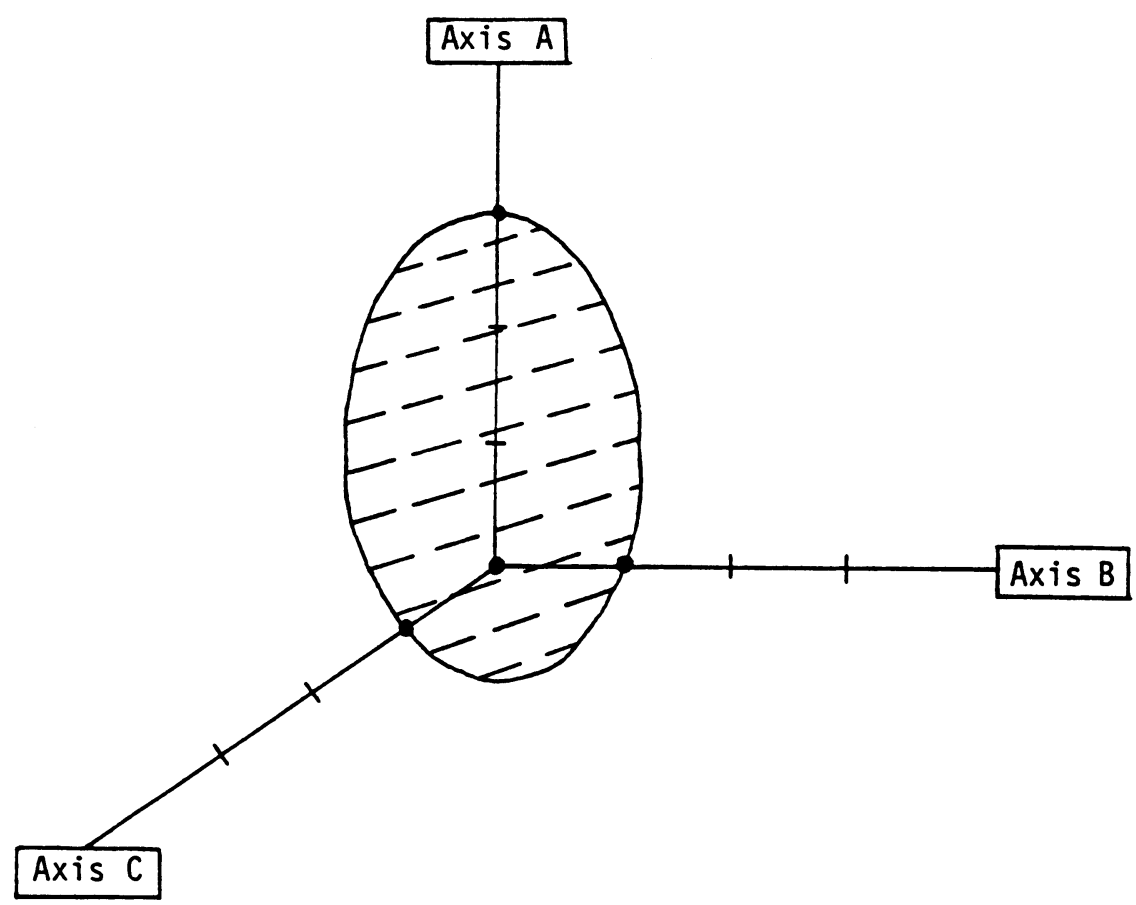

This case is mapped out multidimensionally on Figure 2. The patient shows a severe depressive disorder on Axis A; an obsessional personality organization on Axis B (a mild form of personality disorder); and a relatively stable, resource-rich, help-seeking social milieu on Axis C.

\section{Case \#2}

$\mathrm{Mr} . \mathrm{N}$ is a 23-year old man who appeared in the emergency room of a general hospital in the middle of the night following an impulsive aspirin overdose. He had multiple previous psychiatric contacts for selfdestructive behavior; acute periods of panic, emptiness and dysphoria; 
and periodically abused alcohol and illegal drugs. Unable to work, he lived from day to day on public assistance, odd jobs, and uncertain sources of income. His relationships with others were chaotic and brief, and he had alienated his family and, by now, several hospitals and mental health clinics. Though he repetitively sought help, he held a deep distrust of everyone, particularly psychiatrists, and terminated treatment as soon as his distress abated. A careful history, however, did reveal that he had been experiencing persistent, though minimal, sleep, appetite, and psychomotor symptoms, as well as depressive mood, with increasing hopelessness and suicidal ideation. Family history was positive for impulsivity, alcoholism, sociopathy and depression.

\section{FIGURE 3}

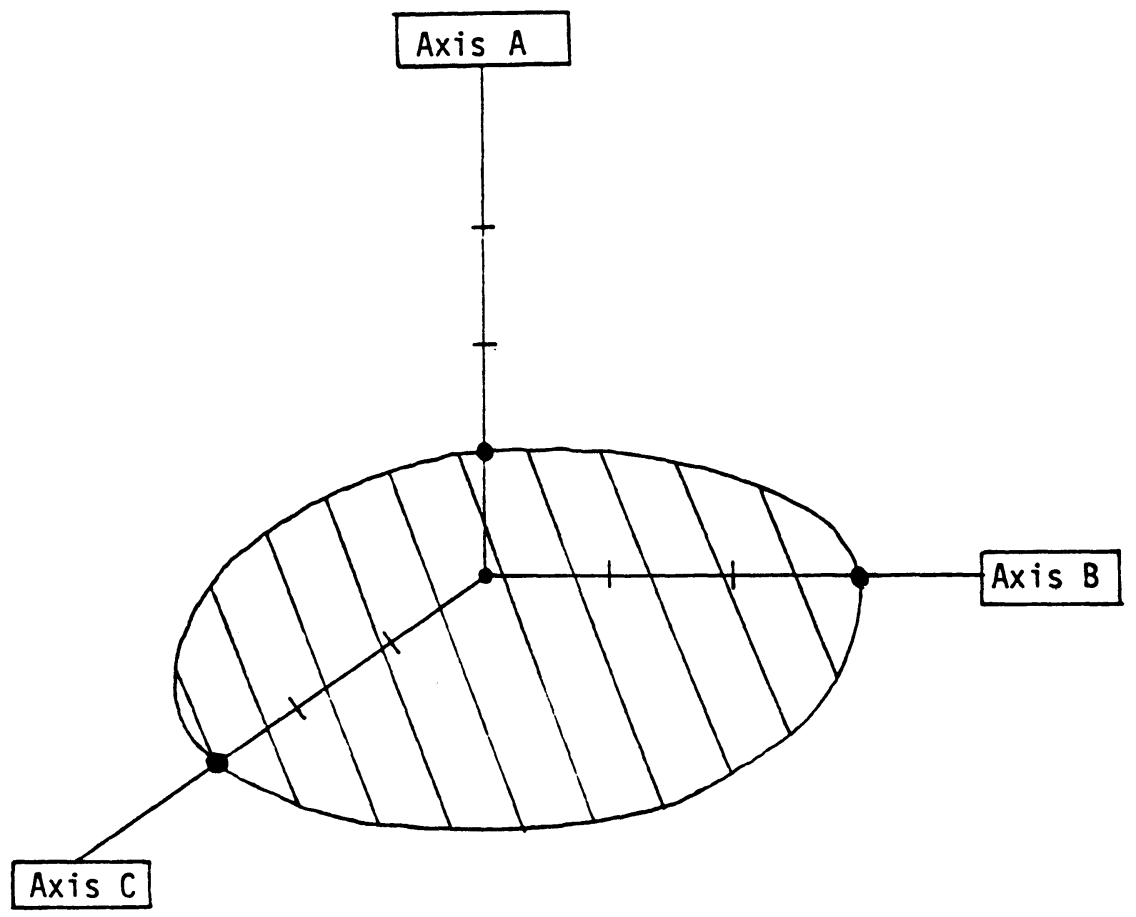


This second case is graphed on Figure 3. Mr. N shows mild depressive symptomatology, as depicted on Axis A. His principal difficulties are, however, psychosocial, and appear such on the other axes. Mr. N evidences the highly maladaptive behavior, dating back to adolescence, that is characteristic of severe character pathology. He also shows profound turmoil and resource depletion in his social context. Axes B and C map and quantify these aspects of his life.

It is by examining Figures 2 and 3 , and by superimposing them (Figure 4), that the value of the biopsychosocial model becomes increasingly clear.

\section{FIGURE 4}

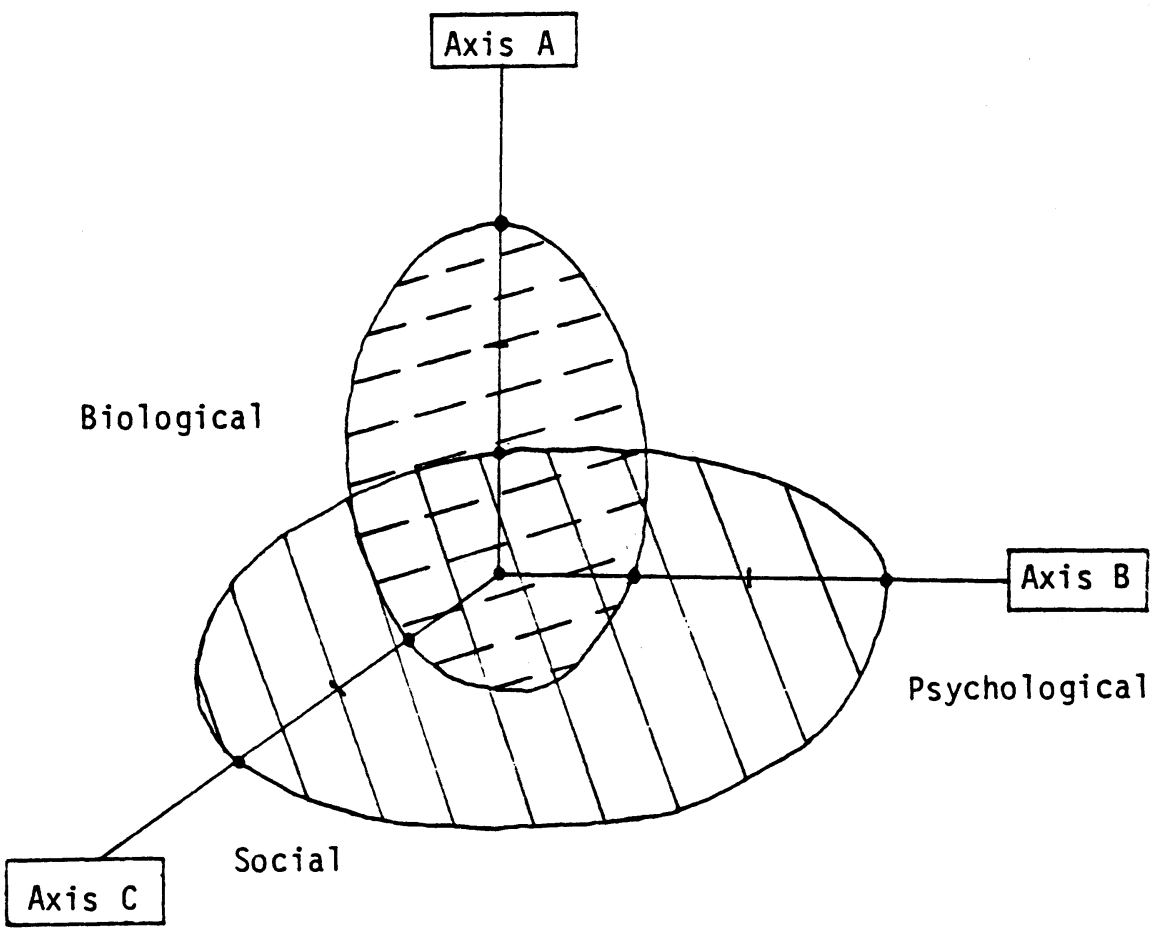


For the patient with major depression as his or her psychiatric disorder, we can see that the diagnosis of this disorder, unidimensionally, is of quite limited clinical utility. The value of the multidimensional model now emerges. The various lenses of the multidimensional biopsychosocial model enable us to see the breadth of the person, not merely his biology, for this is, as these examples demonstrate, too limited a vision.

The model, as applied here, not only gives us breadth; it also focuses our treatment. Treatment specificity surfaces quickly as we view these patients multidimensionally. Dr. M. has a severe, persistent symptomatic disturbance that we know to be responsive to biological interventions. Without biological treatment, Dr. M might not be able to mobilize her ego to begin to reclaim its adaptive abilities. For Dr. M, medications will not interfere with and are likely to complement her psychotherapy. ${ }^{14-16}$ Treatment specificity for Dr. M begins with a careful biological intervention, as the patient's safety is maintained and alliance developed with her and her family. Mr. Mr. N, tricyclic antidepressants would perhaps be minimally beneficial, highly dangerous to this impulsive man, and could draw attention away from the weight of his disturbance, which lies in the psychosocial domain. Instead, our clinical work will be to bring what limited resources may exist in his social field and personality to bear on the crisis at hand.

The biopsychosocial model also enables us to make an informed statement about the course and prognosis of the patient's disease. Dr. M, should she be adequately protected from suicidal behavior, has a very optimistic prognosis and can be expected to improve, with good care, over a period of weeks. The likelihood of improvement with brief treatment would medico-legally support a restrictive environment, if needed, as well as increasingly invasive neurochemical treatment efforts (e.g. from antidepressants to ECT) ${ }^{17,18} \mathrm{Mr}$. $\mathrm{N}$ does not prompt such a sanguine outcome and is not likely to realize any significant lasting improvement in his depressive symptomatology for some time, if at all. For Mr. N, increasingly invasive biological treatments carry more risk than benefit, while involuntary treatment must be approached cautiously because of its regressive pull and the potentially lasting requirement for such control in this man's life.

Finally, for those patients whose treatment eludes us, the biopsychosocial model can serve to direct our research efforts. Accurate diagnosis and identification of the locus of the patient's disturbance offer assistance in shaping research inquiries. Questions of Mr. N's impulsivity will not find sound answers in biological research, nor will Dr. M's severe early morning awakening find resolution in the arena of object relations. 


\section{Conclusion}

The prevailing model directing clinical practice in psychiatry is the biopsychosocial model. Though it does not offer the unity and harmony of a paradigm, its blend of biological, psychological, and social perspectives enables clinicians to view patients multidimensionally, to direct treatment specifically, and to prognosticate accurately. The professionally sanctioned and now extensively used nomenclature of the field (DSM III R) ${ }^{18,20}$ offers linguistic software that fits the hardware of the biopsychosocial model.

Certainly the model has its limitations, as does DSM III R. Such is the nature of any preparadigmatic field and of its evolving nomenclature. Questions of hierarchy of causation, sequence of clinical intervention, and categorical problems between meaning and causation remain.9,21-23 The debate has already begun about how to construct a DSM IV.

In the absence of a paradigm, it is a professional accomplishment to see beyond the confines of any one prescription. Herein lies the utility of the biopsychosocial model. To invoke and apply the model, as we have illustrated in this paper, is to create the conceptual conditions for accurate diagnosis of that which ails the human soul, and then to intervene beneficially, when we can, or to direct specifically our research efforts, when we cannot. To accomplish these tasks is to meet the clinical and ethical mandate of our profession and to feel a sense of personal mastery.

\section{Reference Notes}

1. State of Maryland, Health Claims Arbitration Board, Amended Arbitration, Panel Determination 82-262, Jan. 18, 1984.

2. Rogers vs. Commissioner of Mental Health, 390 Comm. Mass. 489, 1983.

3. Lazare, A.: The Psychiatric examination in the Walk-In clinic: Hypothesis generation and hypothesis testing. Arch. Gen'l Psych 33: 96-102, 1976.

4. Engel, G. L.: The Clinical application of the biopsychosocial model. Am. J. Psych. 137(5): 535-544, 1980.

5. Engel, G. L.: The Biopsychosocial model and the education of health professionals. Ann. NY Acad. Sci. 310: 169-181, 1978.

6. Lazare, A.: Hidden conceptual models in clinical psychiatry. N. Engl. J. Med. 288: 345-351, 1973.

7. Kuhn, T. S.: The Structure of Scientific Revolutions (2nd Edition). Chicago, Univ. of Chicago Press, 1970, p. 10.

8. Watson, R. I.: Psychology: A prescriptive science. In Historical Conceptions of Psychology. Herle, M., Jaynes, J., Sullivan, J. J. (Eds), New York, Springer, 1973. 
9. Sederer, L. I.: The Importance of seeing psychiatry as more than a science. Psychiatric Opinion. Vol. 14, No. 4: 27, 46-47, 1977.

10. Sederer, L. I.: Diagnosis, conceptual models, and the nature of this book. In The Addictions. Milkman, H. B. \& Shaffer, H. J. (Eds), Lexington Books, Lexington, MA, 1985.

11. Kernberg, O. F.: A Psychoanalytic Classification of Character Pathology. J. Am. Psychoanal. Assoc. 18: 800-822, 1970.

12. Kernberg, O. F.: Borderline Conditions and Pathological Narcissism. New York, Jason Aronson, 1975.

13. Vaillant, G. E.: Adaptation to Life. Boston, Little, Brown, 1977.

14. Weissman, M. M., Prusoff, B. A., et al.: The efficacy of drugs and psychotherapy in the treatment of acute depressive episodes. Am. J. Psych. 136: 555-558, 1979.

15. Rounsaville, B. J., Klerman, G. L., et al.: Do psychotherapy and pharmacotherapy for depression conflict? Arch. Gen'l. Psych. 38: 24-29, 1981.

16. Sederer, L. I.: Depression. In Inpatient Psychiatry: Diagnosis and Treatment. Baltimore, MD, Williams \& Wilkins, Ed. 2, 1986, pp. 3-35.

17. Stone, A. A.: Law, Psychiatry and Morality. Washington, DC, Am. Psychiatric Press, 1984.

18. Gutheil, T. G., Appelbaum, P. S.: Clinical Handbook of Psychiatry and The Law. New York, McGraw-Hill, 1982.

19. Spitzer, R. L., Williams, J. B. W., Skodol, A. E.: DSM III: The major achievements and an overview. Am. J. Psych. 136: 815-817, 1979.

20. Klerman, G. L.: The advantages of DSM III. Am. J. Psych. 141(4): 539-542, 1984.

21. Home, H. J.: The concept of mind. Int. J. Psycho-Anal. 47: 42-49, 1966.

22. Abroms, E. M.: Beyond eclecticism. Am. J. Psych. 140: 740-45, 1983.

23. Abroms, E. M.: Psychiatric serialism. Compr. Psych. 22: 372-378, 1981. 